\title{
LES ÉPONYMES DÉMYTHONYMIQUES DANS LE LEXIQUE DU POLONAIS ET DU FRANÇAIS
}

\author{
Artur GAtKOWSKI \\ Université de Łódź
}

\begin{abstract}
The text presents the results of a research on the demythonymic eponyms in Polish and French language, i.e. lexical units, which derive from proper names appearing in the fictional space of classical myths (mythonyms). The primary goal of the study is to determine the cultural code and lexical fields that are represented by demythonymic eponyms. The author also considers the structure of eponyms, distinguishing among them one-element/univerbal and complex/pluriverbal forms. An insight into the onomastic categorization of the demythonymic eponyms allows to establish that in their onymic basis they refer to the proper names of all possible types.
\end{abstract}

\begin{abstract}
À Alicja, pour tous ses gestes amicaux, toute sa gentillesse, la confiance, l'ambiance qu'elle sait créer, les observations pertinentes, la compréhension pour le développement de différents projets et recherches au sein de la Chaire et ensuite de l'Institut d'Études romanes. Chère Alicja, je te félicite pour tous les succès dans ta carrière académique et je te souhaite d'excellents résultats dans ton futur travail ainsi que plein de bonnes choses pour ta vie privée.
\end{abstract}

\section{INTRODUCTION}

La mythologie est inscrite dans la culture universelle comme un des points de repère les plus caractéristiques pour de nombreux concepts qui décrivent tous les espaces ontiques : de l'imaginaire au réel. Elle crée un monde à part, fictif 
et antique, mais par sa réception constante dans l'ère moderne, elle constitue toujours un univers actuel dans lequel se réfléchit la réalité : la vie des hommes, leurs actes, leurs comportements, la vie des animaux et des plantes, les éléments de la nature inanimée, les choses, les événements, les croyances, les idéologies, la philosophie, les arts, la culture dans tous ses aspects civilisationnels, la langue $\mathrm{y}$ incluse.

Il n'est plus possible d'imaginer la civilisation sans (une) mythologie, tant celle classique, surtout grecque et latine, que celle locale, régionale, ethnique, p. ex. hindoue, inca, japonaise ou slave ${ }^{1}$. Chaque mythologie comprise comme l'ensemble des mythes et des légendes sui generis se manifeste dans la culture respective ; en même temps les mythologies se répandent sur d'autres cultures, surtout les cultures avoisinantes, mais aussi plus lointaines qui les absorbent de différentes façons. Dans ce procès, la civilisation occidentale s'avère la plus perceptive. D'une part, elle continue les traditions dont elle est issue, à savoir l'acquis de la mythologie classique gréco-latine et, parallèlement, celui des mythologies « internes » (nationales et européennes). De l'autre, elle puise pour des buts utilitaires dans les mythologies « externes » afin de connaître d'autres coins de la planète, de comprendre leurs histoires, leurs us et coutumes et de s'y confronter s'enrichissant de nouvelles représentations du monde. Il s'agit entre autres du savoir universel encyclopédique que la civilisation des pays occidentaux cherche à emmagasiner et de sa reproduction sous divers points de vue, p. ex. notionnel, scientifique, éducatif, artistique, littéraire, linguistique, etc.

\section{L'OBJET DE L'ÉTUDE}

Dans cette contribution nous nous concentrons sur l'aspect linguistique de 1'opérativité de la culture mythologique, limitée aux témoignages (dé)onymiques ${ }^{2}$ des faits de la mythologie classique des Grecs et des Romains : on prétend qu'elle est la plus caractéristique et représentative pour ce champ d'investigation. Par ailleurs, en utilisant la méthodologie adoptée nous envisageons des recherches plus avancées de l'absorption linguistique pluriculturelle d'autres mythologies que l'on vient de signaler. Cependant, ce qui nous intéresse ici le plus c'est l'apport du code mythologique classique dans la formation et le fonctionnement des éponymes démythonymiques dans le polonais et le français. On peut prétendre que ce type de lexique (dont les bases sont ancrées dans l'onymie mythologique,

\footnotetext{
${ }^{1}$ Voir p. ex. Jacobi (1854); Kempiński (1993).

${ }^{2}$ Soit « déonomastiques » (cf. Schweickard, 1989).
} 
soit la «mythonymie ») $)^{3}$ se caractérise par un haut degré d'internationalisation dont témoignent les deux langues en question autant que plusieurs autres que l'on pourrait prendre ici en considération.

\section{LE PHÉNOMÈNE DE L'ÉPONYMISATION : LE CLASSEMENT ET LES STRUCTURES}

Le phénomène de l'éponymisation implique le recours aux structures propriales qui sont à la base de la formation des éponymes dans les langues ${ }^{4}$. Les éponymes se définissent comme des formes provenant des noms propres qui passent dans la langue générale conformément aux procès de dérivation (entre autres de l'appellativisation, c'est-à-dire du passage du nom propre au nom commun) ${ }^{5}$. Ils désignent des classes d'objets qui ont un lien direct ou indirect avec leur étymon représenté dans ce cas par un onyme. On peut spécifier quelques groupes d'éponymes par rapport à la nature de leur formation, de la construction de l'unité linguistique obtenue et de leur prédestination dans l'usage. La catégorisation qui suit représente un classement des formes éponymiques construites soit à l'aide d'un vocable (éponymes univerbaux) soit composées (phraséologiques - éponymes pluriverbaux).

\subsection{LES ÉPONYMES UNIVERBAUX}

Parmi les éponymes univerbaux on relève :

1. Les lexèmes univerbaux dérivés des onymes, appartenant à différentes catégories grammaticales telles que substantifs, adjectifs, verbes, etc. (p. ex. l'adj. varsovien de l'urbonyme Varsovie, le verbe polonais ocyganić arg. 'mentir, tromper' de l'ethnonyme Cygan 'Gitan') ;

2. Les appellativisations complètes (p. ex. le nom judas de l'anthroponyme Judas ou le nom geberit, lexicalisé comme métonymie de l'expression onymisante « système d'installation ou d'évacuation Geberit » qui s'est réduite à un chrématonyme déanthroponymique appellativisé) ;

${ }^{3} \mathrm{Cf}$. le terme anglais mythonyms (Gałkowski, 2015).

${ }^{4}$ La définition du phénomène linguistique de l'éponymisation, et d'autant plus de l'éponyme comme son résultat lexical, n'est pas claire dans les études linguistiques (voir Konowska, 2008 : 136-142 ; Nakos, 1990/1991 ; Lapierre, 1989). Cf. aussi les doutes au niveau terminologique (onomastique) par rapport au lemme « éponyme » en diverses langues dans l'ICOS Lists of Key Onomastic Terms (https://icosweb.net/drupal/terminology) (dernière consultation : le 20.01.2018).

${ }^{5}$ Pour plus d'informations sur les procès formatifs relatifs aux noms propres en tant que tels et par rapport aux noms communs, voir Caffarelli (2008). Pour le traitement lexicologique et lexicographique des formations déonymiques voir aussi Dereń (2005); Kowalik (2008). 
3. Les éponymes transonymisés (p. ex. le nom polonais jasiek désignant un petit coussin, mais dérivant par la transposition catégorielle de l'hypocoristique anthroponymique Jasiek, diminutif du prénom masculin Jan);

4. Les éponymes occasionnels et ad hoc, souvent chargés de connotations valorisantes : dans le langage de la presse, des médias en général, des jargons, etc. (p. ex. le nom polonais palikotyzacja dérivant du nom du leader d'un des ex-partis politiques polonais Janusz Palikot; le terme signifierait l'état de dégringolade dans la vie publique).

\subsection{LES ÉPONYMES PLURIVERBAUX}

Dans le cadre des formations éponymiques, nous considérons aussi les expressions figées fondées sur un nom propre ou son dérivé qui créent des unités éponymiques pluriverbales ou des phraséologies éponymiques, p. ex. fr. degrés Celsius, pl. skala Celsjusza (la dénomination se réfère au nom de l'inventeur de la mesure) ou le terme culinaire polonais kluski ślaskie ('pâtes silésiennes') qui se réfère par le complément adjectival ślqaskie au coronyme Ślask, la Silésie, suggérant ainsi l'origine et le caractère de la spécialité gastronomique régionale.

Les phraséologies éponymiques sont autant fréquentes que les éponymes univerbaux. Les deux types se croisent : les phraséologies sont fondées sur un éponyme univerbal et les éponymes univerbaux sont obtenus par la réduction, l'élision ou la métonymie des phraséologies éponymiques.

\section{LE CODE MYTHOLOGIQUE COMME SOURCE D'INSPIRATION DANS LE PROCÈS DE L'ÉPONYMISATION}

Les éponymes peuvent être classés aussi par rapport à leur provenance et aux significations culturelles dont ils sont chargés ${ }^{6}$. On note beaucoup de sources culturelles d'éponymes dans la langue. Chaque catégorie onymique et quasiment chaque nom propre sont aptes à servir pour la formation des mots par l'effet de l'éponymisation. L'une de ces sources est constituée par le code mythologique qui abonde en noms propres de tous genres. Il est même plus riche en onymes que d'autres codes qui se déterminent par l'appartenance à un champ donné de la vie, de l'environnement, de l'activité humaine.

${ }^{6}$ Cf. la notion de " charge culturelle partagée » de Robert Galisson (entre autres Galisson, 1988). Cf. aussi la question de la « valeur connotative des noms propres (Rutkowski, 2011). 
Parmi les nomina propria auxquels on attribue l'étiquette terminologique « mythonymes », on trouve maintes dénominations propriales des figures, personnages, animaux, plantes, objets, êtres fantastiques, actes, événements, lieux, édifices, œuvres artisanales et artistiques, idées, histoires et contes mythologiques (les titres des mythes y compris).

Les mythonymes peuvent être rangés parmi : les théonymes, les anthroponymes individuels et collectifs, les dénominations anthroponymisantes, les zoonymes, les phytonymes, les micro- et macrotoponymes (les urbonymes, les ruronymes, les coronymes, les platéonymes, les éconymes, les oronymes, les hydronymes, les nézonymes, etc.), les chrématonymes (les idéonymes, les socioidéonymes, les héortonymes, les éventonymes, etc.) ${ }^{7}$. Il résulte que la typologie dans l'ensemble des onymes mythologiques est plus articulée que la typologie de l'onymie dans la vie réelle. C'est que la mythologie côtoie l'imaginaire et le fictif qui n'ont pas de bornes dans la création des objets de référence et des nominations respectives. L'impression d'abondance dans la masse des mythonymes est aussi due au puisement de l'onymie réelle dans la mythonymie, p. ex. dans la création transonymique intra- et intercatégorielle des noms de personnes à partir des noms des êtres personnifiés, dans la formation des noms de lieux et des chrématonymes de marketing ou idéatifs.

En effet, la mythologie avec ses noms propres se présente comme l'une des sources majeures d'inspiration motivationnelle dans l'onomaturgie contemporaine, p. ex. dans le répertoire des noms d'entreprises, de produits, de marques, de groupes sociaux, d'œuvres d'art ${ }^{8}$. D'où le recours encore plus justifié au phénomène de l'éponymie enracinée dans l'espace démythonymique. Le procès inverse n'est pas observé parce que le monde des mythes classiques une fois fixé est clos et non modifiable ; par conséquent, il n'est plus capable d'intégrer de nouvelles dénominations à moins qu'il ne soit pas volontairement enrichi par les auteurs contemporains, p. ex. pour des raisons de la fiction littéraire.

Il existe encore un autre facteur qui contribue à une impression d'abondance de mythonymes : comme on l'a déjà souligné, ce sont en majeure partie des internationalismes, voir des universalismes qui possèdent leurs adaptations phonétiques et orthographiques dans différentes langues d'accueil. À titre

${ }^{7}$ Pour plus d'informations sur la terminologie onomastique voir Gałkowski (2010) et la bibliographie y incluse.

${ }^{8}$ Voir les noms de marques comme : Apollo (marque de motos, maison de disque), Ariane (un modèle de voiture de la marque Simca), Athena (marque de sous-vêtements, de compagnie d'assurances), Atlas (une bière hollandaise), etc. 
d'exemple, le théonyme polonais Odyseusz dans d'autres langues slaves et baltes se présente comme : bulg. Одисей, serb. Одисеj, ucr. Oдіссей, rus. Одиссей, bielorus. Aдыссей, slove./croa. Odisej, slova./tche. Odysseus, lit. Odiséjas, est. Odysseus, lett. Odisejs, etc.

La reconnaissance des mythonymes par les sujets parlants dépend de leur culture et de leurs besoins communicationnels personnels ; dans la plupart des situations ils ne perçoivent plus les mythonymes transonymisés par la mythologie. Effectivement, le savoir mythologique n'est pas nécessaire à l'utilisation correcte des transonymes démythonymiques. Ceci concerne p. ex. les noms de personnes polonais tels que Apolonia (d'Apollon), Arkadiusz (de l'Arcadie), Astra (d'Astréa, fille de Zeus), Marcin et Marek (de Mars), Saturnin (de Saturne), Trojan (de Troie), etc.

Les transonymes démythonymiques sont démotivés, contrairement aux éponymes démythonymiques qui maintiennent jusqu'aux degrés déterminés leur motivation originaire. Par exemple, il est plus facile d'associer le sens de l'éponyme démythonymique polonais trojan à l'objet qu'il désigne quand on sait que le nom est une dénomination populaire et apparemment chrématonymique équivalente au polonais koń trojański ('cheval de Troie'), utilisé dans le jargon informatif pour désigner un type des virus les plus dangereux pour les ordinateurs. L'éponyme univerbal trojan est le résultat de l'ellipse de l'expression mythologique transonymisée et appellativisée koń trojański qui se réfère au toponyme Troie chargé de signification mythologique particulière. Entre ces unités lexicales il y a un lien connotatif qu'il faut prendre en considération dans la compréhension de l'éponyme. De toute façon, ce lien n'est plus valable dans l'emploi courant du terme trojan qui se libère des associations profondes et fonctionne sans arrière-plan dans son environnement discursif pertinent.

\section{LES ÉPONYMES DÉMYTHONYMIQUES DANS LE SENS LINGUISTIQUE-CULTUREL ET ONOMASTIQUE}

Qu'est-ce que l'on entend exactement par le terme «éponymes démythonymiques »? Ce sont des unités linguistiques lexicalisées, en cours de lexicalisation ou occasionnelles (temporaires, sociolectales ou idiolectales) qui dérivent des mythonymes au sens large du terme. Elles disposent d'un système de références structurelles, sémantiques et pragmatiques que l'on peut associer au monde des mythes, notamment aux noms propres utilisés dans le code mythologique interne et métatextuel. 
Le polonais et le français, comme d'ailleurs toutes les langues de la civilisation occidentale, comportent un important groupe d'éponymes démythonymiques qui est dynamique dans divers registres et au niveau de maints aspects perlocutoires ${ }^{9}$.

\subsection{LA CATÉGORISATION ONOMASTIQUE}

$\mathrm{Du}$ point de vue purement onomastique, les éponymes démythonymiques constituent les formations provenant $\mathrm{des}^{10}$ :

1. Théonymes, p. ex. pl. hermafrodyta 'hermaphrodite', individu bisexué ; le mot dérive de deux théonymes : Hermès, dieu du commerce et messager des dieux, et Aphrodite, déesse de l'amour. Originairement gr. Hermaphroditos désignait l'enfant né de ces deux divinités ;

2. Dénominations anthroponymisantes :

- noms des figures mixtes, mi-hommes, mi-dieux, p. ex. pl. herkules/ Herkules, fr. un hercule, utilisé métaphoriquement pour désigner un homme très fort, vient du nom du demi-dieu Hercule qui dut réaliser ses douze travaux ;

- noms des figures humaines, p. ex. pl. amazonki 'amazones', terme dérivé d'Amazones désignant un peuple de femmes chasseresses et guerrières. Le terme indique par extension les femmes courageuses et cavalières ; aujourd'hui on l'utilise aussi pour parler des femmes dépourvues d'un sein en conséquence des cures oncologiques ;

- noms des monstres, p. ex. mi-hommes, mi-animaux comme le polonais harpia 'harpie' désignant une femme acariâtre ; l'appellativisation des Harpies, créatures à tête de femme et à corps d'oiseau ;

\section{Zoonymes :}

- noms individuels des animaux non altérés, p. ex. pl. Amalteja dans l'expression polonaise róg Amaltei, fr. le corne d'Amalthée, équivalent du polonais róg obfitości, fr. le corne d'abondance, lat. cornu copiae. La nymphe Amalthée fut la nourrice de Zeus. Un jour, la corne d'une chèvre

9 Le corpus que l'on a analysé se compose de soixante-dix formes éponymiques démythonymiques univerbales et d'une vingtaine de phraséologies éponymiques contenant un mythonyme ou son dérivé.

${ }^{10}$ Les significations des termes démythonymiques dans deux sections suivantes sont citées d'après le dictionnaire La mythologie pour origine, accessible en ligne (http://logos.muthos.free.fr/ etymologie/etymytho.htm [dernière consultation : le 19.01.2018]), après avoir été consultées chez Grimal (1999); Kempiński (1993) ; Moormann et Uitterhoeve (2004). 
qui nourrissait Zeus de son lait se cassa. Amalthée la remplit de fleurs et de fruits avant de l'offrir au dieu. Ensuite, Zeus se fit une armure de la peau de cette chèvre : l'égide ;

- noms individuels des animaux altérés, imaginaires, p. ex. pl. feniks, fr. phénix, oiseau fabuleux des déserts de Lybie et d'Éthiopie, de la taille d'un aigle, qui pouvait vivre plusieurs siècles, il ne se reproduisait plus, mais renaissait de ses cendres en s'immolant lui-même sur un bûcher appelé « immortalité »; aujourd'hui on appelle un "phénix » toute personne dotée de qualités exceptionnelles, en quelque sorte unique en son genre ;

- les noms individuels des animaux altérés, imaginaires et personnifiés, p. ex. pl. cerber, fr. cerbère. Ce mot, parfois utilisé pour désigner un gardien intraitable, vient du zoonyme Cerbère, désignant un chien à trois têtes, gardien des enfers ;

4. Toponymes :

- oronymes, p. ex. pl. olimpiada, igrzyska olimpijskie, olimpijski, fr. les olympiades, les jeux olympiques, olympien. Olympe est un massif montagneux au nord de la Grèce, considéré comme la « maison des dieux ». L'adjectif olimpijski, olympien a pris le sens de 'majestueux et serein' (cf. la phraséologie polonaise zachować olimpijski spokój, fr. conserver un calme olympien) ;

- hydronymes, p. ex. pl. letarg, fr. léthargie, tirés du nom du fleuve Léthé situé aux enfers, qui donnait l'oubli à ceux qui s'y baignaient ;

- urbonymes, p. ex. le déjà cité koń trojański, trojan, fr. cheval de Troie, utilisé souvent en anglais : Trojan Horse;

- micro- ou macrotoponymes, p. ex. pl. Pola Elizejskie, fr. Champs-Élysées, utilisés métaphoriquement. Les Champs-Élysées sont la partie des enfers où les ombres des hommes vertueux mènent une existence heureuse et douce;

5. Chrématonymes :

- noms des choses, p. ex. pl. nektar, fr. nectar. À l'origine un nom propre (désignant la boisson des dieux), cet éponyme qualifie aujourd'hui toute boisson délicieuse ;

- noms des fêtes, des jeux, des actions, p. ex. pl. bachanalia, fr. bacchanale. Le nom vient de Bacchantes, nom propre des prêtresses de Bacchus (Dionysos), avant de devenir un nom commun désignant une femme débauchée. Le mot bacchanale est devenu un synonyme d'orgie.

Au-delà des rangements établis, on relève aussi des formes qui se placent à michemin entre les éponymes et les transonymes démythonymiques, p. ex. certains anémonymes (noms des vents), comme le polonais tajfun, fr. typhon, dérivant du 
théonyme Typhon qui désigne une divinité primordiale et un être monstrueux ; le lexème a le sens d'un cyclone tropical très violent. Un autre exemple, c'est le polonais Zefir, zefir, fr. zéphyr. Originairement Zéphyr était le fils d'Éos. Le nom commun zéphire personnifie le vent d'Ouest, agréable ou pluvieux, qui annonce l'arrivée du printemps.

Dans l'interprétation de ce type de faits onomastiques tout dépend de l'attribution catégorielle de la structure obtenue dans le procès formatif. À notre avis, dans le cas du typhon, du zéphyr et des occurrences pareilles, on est près de l'éponymisation.

Par contre, on exclut de la catégorie des éponymes les formes transonymisantes démythonymiques qui nomment des réalités géographiques, p. ex. pl. Atlantyk, fr. Atlantique, dérivant du mythonyme Atlas, ou Amazonka, Amazonia, fr. Amazone, Amazonie, dérivant du mythonyme Amazones.

\subsection{LES CHAMPS LEXICAUX ET LES TERMINOLOGIES}

En tant qu'internationalismes, les éponymes démythonymiques servent à enrichir sémantiquement et pragmatiquement plusieurs champs du lexique et de la terminologie. On pourrait mentionner ici :

- le champ lexical de l'érotisme, de l'amour physique, des comportements sentimentaux, p. ex. les éponymes dérivant du théonyme Éros, dieu de l'amour, comme p. ex. pl. erotyzm, erotyczny, erotyka, erotyzować, erotoman, erotyzacja; pl. afrodyzjak, fr. aphrodisiaque se référant étymologiquement à la déesse de l'amour Aphrodite ;

- le champ lexical de l'aspect extérieur des hommes, des choses, p. ex. pl. adonis, fr. un adonis (Adonis était un héros réputé pour sa beauté, d'où ce mot désignant un jeune homme d'une beauté remarquable); pl. Apollo, apollo, fr. Apollon. Un «Apollon» est un homme d'une beauté parfaite ; pl. chaos, fr. Chaos : le mot désigne un désordre indescriptible ; dans la mythologie c'est le nom du principe originel d'où étaient issus tous les dieux et toutes les choses de la terre ;

- le champ lexical des traits de caractère et de types humains, p. ex. pl. beocki, fr. béotien : le terme vient du coronyme Béotie qui est une région de Grèce dont la capitale est Thèbes. Les grecs considéraient les béotiens comme un peuple lourd d'esprit, d'où le sens actuel de 'peu raffiné, inculte'; pl. Syzyf, fr. Sisyphe : le terme se réfère à l'absurdité d'un travail insurmontable, en vain recommencé. Sisyphe était un roi de Corinthe très ambitieux, cruel et 
avide, il fut condamné par les dieux à être envoyé aux enfers. Indéfiniment, il doit hisser un très lourd rocher en haut d'une montagne, dès qu'il touche le sommet, le rocher retombe en bas de la pente ;

- la terminologie géographique, p. ex. pl. borealny, fr. boréal : le terme signifie 'du nord' (Borée était fils d'un titan et de l'aurore, divinité grecque du vent du nord) ; pl. ocean, fr. océan: Océan était titan et divinité marine, fils du ciel Ouranos et de la Terre Gaia ;

- le champ lexical de la nature et des phénomènes naturels, p. ex. pl. echo, fr. écho : le terme vient d'Écho, nymphe des eaux et des bois; on note ici un lien réciproque entre le vocable originaire gr. echo et son onymisation gr. Echo ;

- la terminologie scientifique, p. ex. pl. geografia, geologia, geomorfizm : ce sont des dérivés du mythonyme Gaia qui dans la cosmogonie grecque personnifiait la terre ; la racine gr. gê est à l'origine de nombreux autres mots en relation avec la terre; pl. hel, fr. hélium : le terme vient d'Hélios, dieu grec du soleil ;

- la terminologie philosophique, idéologique, p. ex. pl. hermetyzm, hermetyczny, fr. hermétisme, hermétique: les termes se réfèrent à l'ensemble des doctrines alchimiques et proviennent du nom du dieu Hermès, assimilé au dieu égyptien Toth à qui on attribue la naissance de l'alchimie ; quant à l'adjectif hermétique 'silencieux, impénétrable', il fait référence au fait qu'Hermès était le grand confident des autres dieux et savait garder les secrets ;

- le champ lexical des comportements individuels et sociaux, p. ex. pl. panika, panikować, fr. panique, paniquer : les éponymes viennent du théonyme Pan, dieu champêtre dont l'apparition subite pouvait être terrifiante, car il était représenté avec des jambes, oreilles et cornes de bouc, de plus, il semait la terreur parmi les nymphes ; pl. higiena, higieniczny, fr. hygiène, hygiénique : les termes proviennent de Hygia, déesse grecque de la santé ;

- la terminologie de médecine, de psychologie, de comportements sociaux, p. ex. pl. hipnoza, hipnotyczny, hipnotyzować, fr. hypnose, hypnotique, hypnotiser : les éponymes dérivent d'Hypnos, nom de la personnification du sommeil, fils d'Érèbe et de Nyx (la nuit) ; pl. morfina, fr. morphine : le terme vient de Morphée, dieu des songes, tout comme l'expression polonaise być w objęciach Morfeusza, fr. être dans les bras de Morphée, qui implique un sommeil profond (Morphée était fils d'Hypnos qui touchait les mortels d'une fleur de pavot et les endormait d'un sommeil plein de rêves); pl. narcyz, narcyzm, narcystyczny, fr. narcisse, narcissisme, narcissique : les éponymes dérivent de Narcisse, beau jeune homme amoureux de sa propre 
image qui fut changé en fleur; pl. kompleks Edypa, fr. le complexe d'Edipe : l'expression est liée au mythe d'CEdipe qui tua son père et épousa sa mère tout comme l'avait prédit l'oracle ; Freud donne ce nom aux tendances instinctives du jeune garçon, attractives à l'égard de sa mère et répulsives à l'égard de son père qu'il considère comme un rival ;

- le champ lexical des arts, p. ex. pl. muzyka, muzyczny, muzykant, fr. musique, musicien : les éponymes viennent du mythonyme Musée(s) qui à son tour dérive du gr. Mouseioni, à savoir un lieu consacré aux Muses, divinités des arts.

Les dix champs lexicaux et terminologiques, établis et illustrés par les exemples mentionnés ci-dessus, semblent les plus pertinents dans l'analyse de la portée sémantico-pragmatique des éponymes démythonymiques. La langue polonaise et la langue française adoptent dans l'usage communicationnel la majeure partie des internationalismes démythonymiques avec leurs significations. On relève toutefois des formes qui ne sont pas passées en polonais ou elles en sont déjà disparues, p. ex. le terme truie noté en français, dérivé du toponyme mythologique Troie par référence au cheval de Troie et désignant la femelle du porc pour le fait de la capacité de son ventre.

\section{Conclusions}

1. La présence des éponymes démythonymiques dans les langues de la civilisation occidentale, parmi lesquelles se trouvent le polonais et le français, est un phénomène bien fondé dans la réalité linguistique des cultures ethniques.

2. Les éponymes démythonymiques continuent à enrichir la langue de formes et de significations pertinentes qui servent à désigner divers référents de la réalité extralinguistique.

3. Les éponymes démythonymiques en tant que structures univerbales ou pluriverbales se présentent sous différentes formes et appartiennent aux catégories grammaticales majeures. Il arrive rarement que le dérivé éponymique univerbal reste une unité inchangeable au niveau structural. Par contre, c'est le cas des éponymes pluriverbaux qui ne sont soumis qu'à la flexion (cf. la déclinaison du polonais : nom. Pola Elizejskie, gén. Pól Elizejskich, dat. Polom Elizejskim, instrum. Polami Elizejskimi, etc.). Les éponymes univerbaux forment le plus souvent des familles de mots avec des noms, des adjectifs, des adverbes, des verbes, p. ex. en polonais dans la famille des dérivés du théonyme Pan on note : panika (subst.), 
paniczny, spanikowany (adj.), panicznie (adv.), panikować, spanikować (v.), panikujacy (part.), etc., ainsi que les formes motivées argotiques, p. ex. les noms panikarz, panikara.

4. Au niveau onomastique, dans les bases des éponymes démythonymiques on trouve tous les types d'onymes qui représentent un ensemble de noms propres fictifs mais très influant sur les langues.

5. Au niveau notionnel et sémantique, les éponymes démythonymiques s'enregistrent dans différentes sphères de la réalité, de l'activité de l'homme et de l'espace mental, ce que démontrent les champs lexicaux et les terminologies variées dans lesquels ces types de lexèmes apparaissent. Il est aussi intéressant d'observer que certains éponymes sont porteurs de plusieurs dénotations et de connotations qui les rendent pluridimensionnels en tant que notions (p. ex. pl. amazonka, fr. amazone, avec des acceptions de 'femme qui monte à cheval', 'prostituée qui racole ses clients en voiture', 'tenue composée d'une jaquette et d'une jupe longue et ample que portaient les femmes pour monter à cheval', 'une espèce de fourmi' et 'différentes espèces de perroquets' ${ }^{11}$.

6. Les éponymes démythonymiques donnent l'accès implicite au code mythologique que les sujets parlants utilisent souvent à leur insu, en confirmant indirectement l'apport de la mythologie et notamment de l'onymie mythologique dans la communication.

\section{Références bibliographiques}

CAFFARELLI, Enzo (2008), “"Un tocco di cipria fuschia per Margherita”. Dal nome proprio al nome comune al nome proprio al nome comune. Spunti per un'analisi dei processi di onimizzazione, lessicalizzazione e transonimizzazione ", in Lessicografia e onomastica 2. Atti delle Giornate internazionali di Studio. Università degli Studi Roma Tre - 14-16 febbraio 2008, Quaderni Internazionali di RION 3 (P. D’Achille, E. Caffarelli éds), Roma, Società Editrice Romana, pp. 115-130.

DEREŃ, Bożena (2005), Pochodne nazw własnych w słowniku i w tekście, Opole, WUO.

GALISSON, Robert (1988), « Cultures et lexicultures. Pour une approche dictionnairique de la culture partagée », Cahiers d'Études Hispaniques Médiévales. Année 7, pp. 325-341.

GAŁKOWSKI, Artur (2010), «Problemi di terminologia onomastica. Contributi per un dibattito », Rivista Italiana di Onomastica, ${ }^{\circ} 2$, pp. 604-624.

${ }^{11}$ Dictionnaire de français Larousse (http://www.larousse.fr/dictionnaires). 
GAŁKOWSKI, Artur (2015), " Mythonyms as the Key to Mythological Phraseology: An Interlinguistic Approach ", in Ancient Myths in the Making of Culture (J. Czerwińska, M. Budzowska éds), Frankfurt am Main-Bern-Bruxelles-New York-Oxford-Warszawa-Wien, Peter Lang, pp. 329-339.

GRIMAL, Pierre (1999), Dictionnaire de la mythologie grecque et romaine, Paris, PUF.

JACOBI, Eduard A. (1854), Dictionnaire mythologique universel ou Biographie mythique des dieux, des personnages fabuleux de la Grèce, de l'Italie, de l'Égypte, de l'Inde, de la Chine, du Japon, de la Scandinavie, de la Gaule, de l'Amérique, de la Polynésie, etc., etc., traduit de l'allemand, refondu et complété par Th. Bernard, Paris, Firmin Didot Frères.

KEMPIŃSKI, Andrzej M. (1993), Stownik mitologii ludów indoeuropejskich, Poznań, SAWW.

KONOWSKA, Agnieszka (2008), Analyse sémantique des éponymes français, Łask, Oficyna Wydawnicza LEKSEM.

KOWALIK, Krystyna (2008), « Pochodne nazw własnych w słownikach ogólnych współczesnej polszczyzny », in Nowe Studia Leksykograficzne 2 (P. Żmigrodzki, R. Przybylska éds), Kraków, Lexis, pp. 191-202.

LAPIERRE, André (1989), « Problématique des éponymes en français contemporain », in Actes du XVIII Congrès International de Linguistique et Philologie Romanes. Université de Trèves 1986. Dialectologie et géographie linguistique (Section VIII) (D. Kremer éd.), vol. IV, Tübingen, Max Niemeyer, pp. 588-597.

MOORMANN, Eric M., UITTERHOEVE, Wilfried (2004), Miti e personaggi del mondo classico: dizionario di storia, letteratura, arte, musica, Milano, Bruno Mondadori.

NAKOS, Dorothy (1990/1991), «Éponymie lexicologique et éponymie terminologique : considérations générales » in ALFA. Actes de Langue Française et de Linguistique. Symposium on French Language \& Linguistics, vol. 3-4, Halifax, Canada, Dalhousie University, pp. 117-125.

RUTKOWSKI, Mariusz (2011), «O zasadach ustalania wartości konotacyjnej nazw własnych », Prace Językoznawcze, t. XIII, pp. 207-214.

SCHWEICKARD, Wolfgang (1989), « Le traitement des formations déonomastiques dans la lexicographie française » in Actes du XVIII ${ }^{e}$ Congrès International de Linguistique et Philologie Romanes. Université de Trèves 1986. Dialectologie et géographie linguistique (Section VIII) (D. Kremer éd.), vol. IV, Tübingen, Max Niemeyer, pp. 242-253.

\section{Sitographie}

www.icosweb.net/drupal/terminology.

www.logos.muthos.free.fr/etymologie/etymytho.htm.

www.larousse.fr/dictionnaires. 